\title{
Male Orgasmic Disorder
}

National Cancer Institute

\section{Source}

National Cancer Institute. Male Orgasmic Disorder. NCI Thesaurus. Code C34959.

Persistent delay or absence in orgasm not accounted for by a medical reason. 